\title{
Perturbative Expansion Technique for Non-linear FBSDEs with Interacting Particle Method *
}

\author{
Masaaki Fujii $†$ Akihiko Takahashi ${ }^{\ddagger}$ \\ First version: April 12, 2012 \\ Current version: April 23, 2012
}

\begin{abstract}
In this paper, we propose an efficient Monte Carlo implementation of non-linear FBSDEs as a system of interacting particles inspired by the ideas of branching diffusion method. It will be particularly useful to investigate large and complex systems, and hence it is a good complement of our previous work presenting an analytical perturbation procedure for generic non-linear FBSDEs. There appear multiple species of particles, where the first one follows the diffusion of the original underlying state, and the others the Malliavin derivatives with a grading structure. The number of branching points are capped by the order of perturbation, which is expected to make the scheme less numerically intensive. The proposed method can be applied to semi-linear problems, such as American and Bermudan options, Credit Value Adjustment (CVA), and even fully non-linear issues, such as the optimal portfolio problems in incomplete and/or constrained markets, feedbacks from large investors, and also the analysis of various risk measures.
\end{abstract}

Keywords : BSDE, FBSDE, Asymptotic Expansion, Malliavin Derivative, interacting particle method, branching diffusion

\footnotetext{
${ }^{*}$ This research is supported by CARF (Center for Advanced Research in Finance) and the global COE program "The research and training center for new development in mathematics." All the contents expressed in this research are solely those of the authors and do not represent any views or opinions of any institutions. The authors are not responsible or liable in any manner for any losses and/or damages caused by the use of any contents in this research.

${ }^{\dagger}$ Graduate School of Economics, The University of Tokyo

${ }^{\ddagger}$ Graduate School of Economics, The University of Tokyo
} 


\section{Introduction}

The forward backward stochastic differential equations (FBSDEs) were first introduced by Bismut (1973) [1], and then later extended by Pardoux and Peng (1990) [27] for general non-linear cases. They were found particularly relevant for optimal portfolio and indifference pricing issues in incomplete and/or constrained markets. Their financial applications are discussed in details in, for example, El Karoui, Peng and Quenez (1997a) [10, Ma and Yong (2000) [24] and a recent book edited by Carmona (2009) [4]. The importance of FBSDEs will increase in coming years even among practitioners where the new financial regulations will put significant constraints on available assets and trading strategies.

In the recent paper, Fujii \& Takahashi (2011) [12 proposed a new perturbative solution technique for generic non-linear FBSDEs. It was shown that a non-linear FBSDE can be decomposed into a series of linear and decoupled FBSDEs by treating a non-linear driver and feedback terms as perturbations to the corresponding decoupled free system. In particular, it allows analytical explicit expressions for the backward components with the help of the asymptotic expansion technique (See, for example [28, 21, 30, 29].). A backward component of the diffusion part was shown to be obtained by directly considering dynamics of the stochastic flow, which denotes a Malliavin derivative of the underling state process, or simply applying Itô formula to the result of the other part. In Fujii \& Takahashi (2012) [13, the method was applied to a quadratic-growth FBSDE appearing in an incomplete financial market with stochastic volatility. Explicit expressions for both of the backward components were obtained up to the third order of the volatility of volatility. The comparison to the exact solution with Cole-Hopf transformation demonstrated effectiveness of the perturbative expansion.

Notice the fact that one can already apply standard Monte Carlo simulation to the results obtained in each order of the perturbative expansion in [12]. However, due to its convoluted nature, it contains multi-dimensional time integrations of expectation values which make the naive applications too time consuming, particularly for the evaluation of higher order perturbation terms. To handle this problem, we applied the idea of particle representation used in branching diffusion models, such as in McKean (1975) [26]. There, the convoluted expectation is compressed into a single standard expectation by introducing an intensity of the particle interaction. McKean [26] applied the method to solve a particular type of semi-linear PDE, where a single particle splits into two at each interaction time and creates a cascade of the identical particles. Note that, our method is not directly related to McKean [26] since the interested system is already decomposed into a set of linear problems, although we have used the similar particle representation to avoid nested simulations.

The analysis of branching Markov process and related problems in semi-linear PDEs has a long history. Some of the well-known works are Fujita (1966) [15], Ikeda, Nagasawa \& Watanabe $(1965,1966,1968)$ [17, 18, 19], Ikeda et.al. $(1996,1997)$ [20] and Nagasawa \& Sirao (1969) [25]. As for a recent work, in particular, Chakraborty \& LópezMimbela (2008) used particle representation where the number of offspring at each interaction point is randomly drawn by some probability distribution, which can be finitely many or infinite. The authors used the branching particle representation 11 to study the existence of global solutions for semi-linear PDEs with a non-linear driver given by a

\footnotetext{
${ }^{1}$ The same branching representation is already seen in 19, for example.
} 
generic polynomial function 2. Recently, Henry-Labordère (2012) [16] introduced a particle representation to study the semi-linear problems in finance. He called it marked branching diffusion and has discussed its application to efficiently calculate CVA (credit value adjustment) in one-shot Monte Carlo simulation. He also referred to its application to other semi-linear problems, such as American options, as well as its possible extension to truly non-linear problems by using Malliavin derivatives.

In the current paper, we combine the idea of particle representation and the perturbation technique developed in the previous work [12. We provide a straightforward simulation scheme to solve fully-nonlinear decoupled as well as coupled FBSDEs at each order of perturbative approximation. In contrast to the direct application of branching diffusion method, the number of branching points are capped by the order of perturbative expansion, which is due to the linearity of the decomposed FBSDE system. This property is expected to make Monte Carlo simulation less numerically intensive. Our method can be applied to semi-linear problems, such as American and Bermudan options 3 , Credit Value Adjustment (CVA) as special examples. It can be also applied to fully non-linear (and fully coupled) issues, such as the optimal portfolio problems in incomplete and/or constrained market, analysis for various risk measures as well as for the feedbacks from so-called large investors. Concrete applications of the new method will be published separately [14].

\section{Setup}

We first consider generic decoupled non-linear FBSDEs. Let us use the same setup assumed in the work [12. The probability space is taken as $(\Omega, \mathcal{F}, P)$ and $T \in(0, \infty)$ denotes some fixed time horizon. $W_{t}=\left(W_{t}^{1}, \cdots, W_{t}^{r}\right)^{*}, 0 \leq t \leq T$ is $\mathbb{R}^{r}$-valued Brownian motion defined on $(\Omega, \mathcal{F}, P)$, and $\left(\mathcal{F}_{t}\right)_{\{0 \leq t \leq T\}}$ stands for P-augmented natural filtration generated by the Brownian motion.

We consider the following forward-backward stochastic differential equation (FBSDE)

$$
\begin{aligned}
d V_{s} & =-f\left(X_{s}, V_{s}, Z_{s}\right) d s+Z_{s} \cdot d W_{s} \\
V_{T} & =\Psi\left(X_{T}\right)
\end{aligned}
$$

where $V$ takes the value in $\mathbb{R}$, and $X_{t} \in \mathbb{R}^{d}$ is assumed to follow a generic Markovian forward SDE

$$
d X_{s}=\gamma_{0}\left(X_{s}\right) d s+\gamma\left(X_{s}\right) \cdot d W_{s} .
$$

Here, we absorbed an explicit dependence on time to $X$ by allowing some of its components can be a time itself. $\Psi\left(X_{T}\right)$ denotes the terminal payoff where $\Psi(x)$ is a deterministic function of $x$. The following approximation procedures can be applied in the same way also in the presence of coupon payments. $Z$ and $\gamma$ take values in $\mathbb{R}^{r}$ and $\mathbb{R}^{d \times r}$ respectively, and "." in front of the $d W$ represents the summation for the components of $r$-dimensional

\footnotetext{
${ }^{2}$ For recent developments and reviews of the particle methods, see for examples [8, 9]. There exist a significant amount of works related to branching diffusion in 1960's and 70's. There are also a vast range of new applications and enhancements in biology, such as gene mutation and population growth problems, as well as in engineering issues. We have not yet obtained the whole picture of research history related to branching diffusion and are welcoming information from those familiar with the topic.

${ }^{3}$ A BSDE formulation for an American option was shown in El Karoui etal. (1997b) 11, which was recently studied by Labart \& Lelong (2011) [22] based on regression based Monte Carlo simulation.
} 
Brownian motion. Throughout this paper, we are going to assume that the appropriate regularity conditions are satisfied for the necessary treatments.

Let us fix the initial time as $t$. We denote the Malliavin derivative of $X_{u}(u \geq t)$ at time $t$ as

$$
\mathcal{D}_{t} X_{u} \in \mathbb{R}^{r \times d}
$$

Its dynamics in terms of the future time $u$ is specified by the well-known stochastic flow:

$$
\begin{aligned}
d\left(Y_{t, u}\right)_{j}^{i} & =\partial_{k} \gamma_{0}^{i}\left(X_{u}\right)\left(Y_{t u}\right)_{j}^{k} d u+\partial_{k} \gamma_{a}^{i}\left(X_{u}\right)\left(Y_{t u}\right)_{j}^{k} d W_{u}^{a} \\
\left(Y_{t, t}\right)_{j}^{i} & =\delta_{j}^{i}
\end{aligned}
$$

where $\partial_{k}$ denotes the differential with respect to the k-th component of $X$, and $\delta_{j}^{i}$ denotes Kronecker delta. Here, $i$ and $j$ run through $\{1, \cdots, d\}$ and $\{1, \cdots, r\}$ for $a$. Throughout the paper, we adopt Einstein notation which assumes the summation of all the paired indexes. Using the known chain rule of Malliavin derivative, one sees

$$
\left(\mathcal{D}_{t} X_{u}^{i}\right)=\int_{t}^{u} \partial_{k} \gamma_{0}^{i}\left(X_{s}\right)\left(\mathcal{D}_{t} X_{s}^{k}\right) d s+\int_{t}^{u} \partial_{k} \gamma^{i}\left(X_{s}\right)\left(\mathcal{D}_{t} X_{s}^{k}\right) \cdot d W_{s}+\gamma^{i}\left(X_{t}\right)
$$

and hence it satisfies

$$
\left(\mathcal{D}_{t} X_{u}^{i}\right)_{a}=\left(Y_{t, u}\right)_{j}^{i} \gamma_{a}^{j}\left(X_{t}\right)=\left(Y_{t, u} \gamma\left(X_{t}\right)\right)_{a}^{i}
$$

where " $a "$ is the index of $r$-dimensional Brownian motion.

\section{Expansion into a series of Linear FBSDE System}

Following the perturbative method proposed in [12], let us introduce the perturbation parameter $\epsilon$ and then write the equation as

$$
\left\{\begin{array}{l}
d V_{s}^{(\epsilon)}=-\epsilon f\left(X_{s}, V_{s}^{(\epsilon)}, Z_{s}^{(\epsilon)}\right) d s+Z_{s}^{(\epsilon)} \cdot d W_{s} \\
V_{T}^{(\epsilon)}=\Psi\left(X_{T}\right)
\end{array}\right.
$$

where $\epsilon=1$ corresponds to the original model 4 . We suppose that the solution can be expanded in a power series of $\epsilon$ :

$$
\begin{aligned}
& V_{t}^{(\epsilon)}=V_{t}^{(0)}+\epsilon V_{t}^{(1)}+\epsilon^{2} V_{t}^{(2)}+\epsilon^{3} V_{t}^{(3)}+\cdots \\
& Z_{t}^{(\epsilon)}=Z_{t}^{(0)}+\epsilon Z_{t}^{(1)}+\epsilon^{2} Z_{t}^{(2)}+\epsilon^{3} Z_{t}^{(3)}+\cdots
\end{aligned}
$$

If the non-linearity is sub-dominant, one can expect to obtain reasonable approximation of the original system by putting $\epsilon=1$ at the end of calculation.

The dynamics of each pair $\left(V^{(i)}, Z^{(i)}\right)$ can be easily derived as follows:

\section{Zero-th order}

$$
\left\{\begin{array}{l}
d V_{s}^{(0)}=Z_{s}^{(0)} \cdot d W_{s} \\
V_{T}^{(0)}=\Psi\left(X_{T}\right)
\end{array}\right.
$$

\footnotetext{
${ }^{4}$ It is possible to extract the linear term from the driver and treat separately. Here, we simply leave it in a driver, or work in a "discounted" base to remove linear term in $V$.
} 
First order

$$
\left\{\begin{array}{l}
d V_{s}^{(1)}=-f\left(X_{s}, V_{s}^{(0)}, Z_{s}^{(0)}\right) d s+Z_{s}^{(1)} \cdot d W_{s} \\
V_{T}^{(1)}=0
\end{array}\right.
$$

Second order

$$
\left\{\begin{array}{l}
d V_{s}^{(2)}=-\left\{V_{s}^{(1)} \frac{\partial}{\partial v}+\left(Z_{s}^{a(1)}\right) \frac{\partial}{\partial z^{a}}\right\} f\left(X_{s}, V_{s}^{(0)}, Z_{s}^{(0)}\right) d s+Z_{s}^{(2)} \cdot d W_{s} \\
V_{T}^{(2)}=0
\end{array}\right.
$$

\section{Third order}

$$
\left\{\begin{aligned}
d V_{s}^{(3)}= & -\left\{V_{s}^{(2)} \frac{\partial}{\partial v}+Z_{s}^{2(a)} \frac{\partial}{\partial z^{a}}+\frac{1}{2}\left(V_{s}^{(1)}\right)^{2} \frac{\partial^{2}}{\partial v^{2}}+V_{s}^{(1)} Z_{s}^{a(1)} \frac{\partial^{2}}{\partial v \partial z^{a}}\right. \\
& \left.+\frac{1}{2} Z_{s}^{a(1)} Z_{s}^{b(1)} \frac{\partial^{2}}{\partial z^{a} \partial z^{b}}\right\} f\left(X_{s}, V_{s}^{(0)}, Z_{s}^{(0)}\right) d s+Z_{s}^{(3)} \cdot d W_{s} \\
V_{T}^{(3)}=0 &
\end{aligned}\right.
$$

One can continue to an arbitrary higher order in the same way.

Note that the higher order backward components $\left(V^{(n)}, Z^{(n)}\right)_{\{n \geq 1\}}$ are always outside of the non-linear functions. This property arises naturally due to the very nature of perturbation. As we shall see, this is crucial to suppress the number of particles in the numerical simulation.

\section{Interacting Particle Interpretation}

Let us fix the initial time $t$ and set $X_{t}=x_{t}$.

\section{$4.1 \quad \epsilon-0$ th Order}

For the zeroth order, it is easy to see

$$
\begin{aligned}
V_{t}^{(0)} & =\mathbb{E}\left[\Psi\left(X_{T}\right) \mid \mathcal{F}_{t}\right] \\
Z_{t}^{a(0)} & =\mathbb{E}\left[\partial_{i} \Psi\left(X_{T}\right)\left(\mathcal{D}_{t}^{a} X_{T}^{i}\right) \mid \mathcal{F}_{t}\right] \\
& =\mathbb{E}\left[\partial_{i} \Psi\left(X_{T}\right)\left(Y_{t T} \gamma\left(X_{t}\right)\right)_{a}^{i} \mid \mathcal{F}_{t}\right]
\end{aligned}
$$

It is clear that they can be evaluated by standard Monte Carlo simulation. However, for their use in higher order approximation, it is crucial to obtain explicit approximate expressions for these two quantities. As proposed in [12], we use asymptotic expansion technique [28, 21, 30, 29] for this purpose. When $\Psi$ is a smooth function, it is quite straightforward. Even if $\Psi$ is not a smooth function, such as an option payoff, one can obtain explicit expressions of $\left(V^{(0)}, Z^{(0)}\right)$ in terms of $X_{t}$, too. This is because, one can derive an approximate joint transition density of general diffusion processes by the asymptotic 
expansion 5. In the following, let us suppose that we have obtained the solutions up to a given order of asymptotic expansion, and write each of them as a function of $x_{t}$ :

$$
\left\{\begin{array}{l}
V_{t}^{(0)}=v^{(0)}\left(x_{t}\right) \\
Z_{t}^{(0)}=z^{(0)}\left(x_{t}\right)
\end{array}\right.
$$

\section{$4.2 \epsilon-1$ st Order}

Since the BSDE is linear, we can integrate as before. Here, let us first consider the evaluation of $V_{t}^{(1)}$.

$$
\begin{aligned}
V_{t}^{(1)} & =\int_{t}^{T} \mathbb{E}\left[f\left(X_{u}, V_{u}^{(0)}, Z_{u}^{(0)}\right) \mid \mathcal{F}_{t}\right] d u \\
& =\int_{t}^{T} \mathbb{E}\left[f\left(X_{u}, v^{(0)}\left(X_{u}\right), z^{(0)}\left(X_{u}\right)\right) \mid \mathcal{F}_{t}\right] d u
\end{aligned}
$$

Although it is possible to carry out standard Monte Carlo simulation for every time $u \in$ $(t, T)$ and integrate to obtain the $V_{t}^{(1)}$, the time integration becomes numerically quite heavy. In fact, it will soon become infeasible for $\epsilon$ higher order terms that include multidimensional integration of time. We now introduce particle interpretation by McKean [26] developed for the study of semilinear PDEs:

Proposition 1 The $V_{t}^{(1)}$ in (4.4) can be equivalently expressed as

$$
V_{t}^{(1)}=\mathbf{1}_{\{\tau>t\}} \mathbb{E}\left[\mathbf{1}_{\{\tau<T\}} \hat{f}_{t}\left(X_{\tau}, v^{(0)}\left(X_{\tau}\right), z^{(0)}\left(X_{\tau}\right)\right) \mid \mathcal{F}_{t}\right]
$$

Here $\tau$ is the time of interaction which is drawn independently from Poisson distribution with an arbitrary deterministic positive intensity process $\lambda_{t}$. It can be a positive constant for the simplest case. $6 \hat{f}$ is defined as

$$
\hat{f}_{t}\left(x, v^{(0)}(x), z^{(0)}(x)\right):=\frac{1}{\lambda_{s}} e^{\int_{t}^{s} \lambda_{u} d u} f\left(x, v^{(0)}(x), z^{(0)}(x)\right) .
$$

Proof: Define the new process for $(s>t)$ :

$$
\hat{V}_{t, s}^{(1)}=e^{\int_{t}^{s} \lambda_{u} d u} V_{s}^{(1)}
$$

then its dynamics is given by

$$
\begin{aligned}
d \hat{V}_{t, s}^{(1)} & =e^{\int_{t}^{s} \lambda_{u} d u}\left\{\lambda_{s} V_{s}^{(1)} d s-f\left(X_{s}, v^{(0)}\left(X_{s}\right), z^{(0)}\left(X_{s}\right)\right) d s+Z_{s}^{(1)} \cdot d W_{s}\right\} \\
& =\lambda_{s} \hat{V}_{t, s}^{(1)} d s-\lambda_{s} \hat{f}_{t}\left(X_{s}, v^{(0)}\left(X_{s}\right), z^{(0)}\left(X_{s}\right)\right) d s+e^{\int_{t}^{s} \lambda_{u} d u} Z_{s}^{(1)} \cdot d W_{s} .
\end{aligned}
$$

Since we have $\hat{V}_{t, t}^{(1)}=V_{t}^{(1)}$, one can easily see the following relation holds:

$$
V_{t}^{(1)}=\int_{t}^{T} \mathbb{E}\left[e^{-\int_{t}^{u} \lambda_{s} d s} \lambda_{u} \hat{f}_{t}\left(X_{u}, v^{(0)}\left(X_{u}\right), z^{(0)}\left(X_{u}\right)\right) \mid \mathcal{F}_{t}\right] d u
$$

\footnotetext{
${ }^{5}$ We intend to use the result of asymptotic expansion only for higher order approximations.

${ }^{6} \mathrm{It}$ is not difficult to make it a stochastic process.
} 
It is clear for those familiar with credit risk modeling [2, 3], it is nothing but the present value of default payment where the default intensity is $\lambda$ with the default payoff at $s(>t)$ as $\hat{f}_{t}\left(X_{s}, v^{(0)}\left(X_{s}\right), z^{(0)}\left(X_{s}\right)\right)$. Thus, it is clear that (4.9) is equivalent to (4.5).

Now, let us consider the martingale component $Z^{(1)}$. It can be expressed as

$$
Z_{t}^{(1)}=\int_{t}^{T} \mathbb{E}\left[\mathcal{D}_{t} f\left(X_{u}, v^{(0)}\left(X_{u}\right), z^{(0)}\left(X_{u}\right)\right) \mid \mathcal{F}_{t}\right] d u
$$

We perform the similar transformation for $Z^{(1)}$ to make it easier to interpret in the interacting particle model. Firstly, let us observe that the dynamics of Malliavin derivative of $V^{(1)}$ follows

$$
\begin{aligned}
d\left(\mathcal{D}_{t} V_{s}^{(1)}\right)= & -\left(\mathcal{D}_{t} X_{s}^{i}\right)\left\{\partial_{i}+\partial_{i} v^{(0)}\left(X_{s}\right) \partial_{v}+\partial_{i} z^{a(0)}\left(X_{s}\right) \partial_{z^{a}}\right\} f\left(X_{s}, v^{(0)}\left(X_{s}\right), z^{(0)}\left(X_{s}\right)\right) d s \\
& +\left(\mathcal{D}_{t} Z_{s}^{(1)}\right) \cdot d W_{s} \\
\mathcal{D}_{t} V_{t}^{(1)}= & Z_{t}^{(1)}
\end{aligned}
$$

For lighten the notation, let us introduce a derivative operator

$$
\nabla_{i}\left(x, v^{(0)}, z^{(0)}\right)=\partial_{i}+\partial_{i} v^{(0)}(x) \partial_{v}+\partial_{i} z^{a(0)}(x) \partial_{z^{a}}
$$

and also

$$
f\left(x, v^{(0)}, z^{(0)}\right) \equiv f\left(x, v^{(0)}(x), z^{(0)}(x)\right)
$$

Now, we can write Eq. (4.12) as

$$
d\left(\mathcal{D}_{t} V_{s}^{(1)}\right)=-\left(\mathcal{D}_{t} X_{s}^{i}\right) \nabla_{i}\left(X_{s}, v^{(0)}, z^{(0)}\right) f\left(X_{s}, v^{(0)}, z^{(0)}\right) d s+\left(\mathcal{D}_{t} Z_{s}^{(1)}\right) \cdot d W_{s}
$$

Define, for $(s>t)$,

$$
\widehat{\mathcal{D}_{t} V_{s}^{(1)}}=e^{\int_{t}^{s} \lambda_{u} d u}\left(\mathcal{D}_{t} V_{s}^{(1)}\right)
$$

then its dynamics can be written as

$$
\begin{aligned}
& d\left(\widehat{\mathcal{D}_{t} V_{s}^{(1)}}\right)=e^{\int_{t}^{s} \lambda_{u} d u}\left\{\lambda_{s}\left(\mathcal{D}_{t} V_{s}^{(1)}\right) d s-\left(\mathcal{D}_{t} X_{s}^{i}\right) \nabla_{i}\left(X_{s}, v^{(0)}, z^{(0)}\right) f\left(X_{s}, v^{(0)}, z^{(0)}\right) d s\right. \\
& \left.\quad+\mathcal{D}_{t} Z_{s}^{(0)} \cdot d W_{s}\right\} \\
& =\lambda_{s}\left(\widehat{\mathcal{D}_{t} V_{s}^{(1)}}\right) d s-\lambda_{s}\left(\mathcal{D}_{t} X_{s}^{i}\right) \nabla_{i}\left(X_{s}, v^{(0)}, z^{(0)}\right) \hat{f}_{t}\left(X_{s}, v^{(0)}, z^{(0)}\right) d s \\
& \quad+e^{\int_{t}^{s} \lambda_{u} d u}\left(\mathcal{D}_{t} Z_{s}^{(0)}\right) \cdot d W_{s}
\end{aligned}
$$

We have

$$
\widehat{\mathcal{D}_{t} V_{t}^{(1)}}=Z_{t}^{(1)}
$$

and hence

$$
Z_{t}^{(1)}=\int_{t}^{T} \mathbb{E}\left[e^{-\int_{t}^{u} \lambda_{s} d s} \lambda_{s}\left(\mathcal{D}_{t} X_{u}^{i}\right) \nabla_{i}\left(X_{u}, v^{(0)}, z^{(0)}\right) \hat{f}_{t}\left(X_{u}, v^{(0)}, z^{(0)}\right) \mid \mathcal{F}_{t}\right]
$$

Thus, following the same argument of the proposition 1, we can conclude: 
Proposition $2 Z_{t}^{(1)}$ in 4.10 is equivalently expressed as

$$
Z_{t}^{a(1)}=\mathbf{1}_{\{\tau>t\}} \mathbb{E}\left[\mathbf{1}_{\{\tau<T\}}\left(Y_{t, \tau} \gamma\left(X_{t}\right)\right)_{a}^{i} \nabla_{i}\left(X_{\tau}, v^{(0)}, z^{(0)}\right) \hat{f}_{t}\left(X_{\tau}, v^{(0)}, z^{(0)}\right) \mid \mathcal{F}_{t}\right]
$$

where the definitions of random time $\tau$ and the intensity process $\lambda$ are the same as those in proposition 1 .

As we shall see later, interpreting $(X, Y)$ as a pair of particles allows an efficient Monte Carlo implementation. For the evaluation of $Z^{(1)}$ for example, one can consider it as an system of two particles $(X, Y)$, which have the intensity $\lambda$ of the interaction that produces

$$
\left(Y_{t \tau} \gamma\left(X_{t}\right)\right)_{a}^{i} \nabla_{i}\left(X_{\tau}, v^{(0)}, z^{(0)}\right) \hat{f}_{t}\left(X_{\tau}, v^{(0)}, z^{(0)}\right)
$$

at the interaction point and annihilate altogether. For $V^{(1)}$, the interpretation is much simpler. A single particle $X$ with the decay rate of $\lambda$ leaves $\hat{f}$ at its decay point and vanishes.

\section{$4.3 \quad \epsilon$-2nd Order}

For the $\epsilon$-2nd order, one can observe that

$$
\begin{aligned}
V_{t}^{(2)} & =\int_{t}^{T} \mathbb{E}\left[\left(V_{u}^{(1)} \partial_{v}+Z_{u}^{a(1)} \partial_{z^{a}}\right) f\left(X_{u}, v^{(0)}, z^{(0)}\right) \mid \mathcal{F}_{t}\right] d u \\
Z_{t}^{(2)} & =\int_{t}^{T} \mathbb{E}\left[\mathcal{D}_{t}\left\{\left(V_{u}^{(1)} \partial_{v}+Z_{u}^{a(1)} \partial_{z^{a}}\right) f\left(X_{u}, v^{(0)}, z^{(0)}\right)\right\} \mid \mathcal{F}_{t}\right] d u
\end{aligned}
$$

solve the BSDE (3.6). Its particle interpretation is available by the similar transformation.

Firstly, for $(s>t)$, let us define

$$
\hat{V}_{t, s}^{(2)}=e^{\int_{t}^{s} \lambda_{u} d u} V_{s}^{(2)}
$$

with some appropriate intensity process $\lambda$. Then it follows

$$
\begin{gathered}
d \hat{V}_{t, s}^{(2)}=\quad \lambda_{s} \hat{V}_{t, s}^{(2)} d s-\lambda_{s}\left(V_{s}^{(1)} \partial_{v}+Z_{s}^{a(1)} \partial_{z^{a}}\right) \hat{f}_{t}\left(X_{s}, v^{(0)}, z^{(0)}\right) d s \\
+e^{\int_{t}^{s} \lambda_{u} d u} Z_{s}^{(2)} \cdot d W_{s}
\end{gathered}
$$

Observing that $\hat{V}_{t, t}^{(2)}=V_{t}^{(2)}$, one can confirm that

$$
V_{t}^{(2)}=\mathbf{1}_{\left\{\tau_{1}>t\right\}} \mathbb{E}\left[\mathbf{1}_{\left\{\tau_{1}<T\right\}}\left(V_{\tau_{1}}^{(1)} \partial_{v}+Z_{\tau_{1}}^{a(1)} \partial_{z^{a}}\right) \hat{f}_{t}\left(X_{\tau_{1}}, v^{(0)}, z^{(0)}\right) \mid \mathcal{F}_{t}\right]
$$

where $\tau_{1}$ is the random interaction time with intensity $\lambda$. Now, using the tower property of conditional expectations, one can conclude that

Proposition $3 V_{t}^{(2)}$ in 4.21) is equivalently expressed as

$$
\begin{gathered}
V_{t}^{(2)}=\mathbf{1}_{\left\{\tau_{1}>t\right\}} \mathbb{E}\left[\mathbf{1}_{\left\{\tau_{1}<\tau_{2}<T\right\}}\left(\partial_{v} \hat{f}_{t, \tau_{1}}\right) \hat{f}_{\tau_{1}, \tau_{2}} \mid \mathcal{F}_{t}\right] \\
+\mathbf{1}_{\left\{\tau_{1}>t\right\}} \mathbb{E}\left[\mathbf{1}_{\left\{\tau_{1}<\tau_{2}<T\right\}} \partial_{z^{a}} \hat{f}_{t, \tau_{1}}\left(Y_{\tau_{1}, \tau_{2}} \gamma_{\tau_{1}}\right)_{a}^{i} \nabla_{i, \tau_{2}} \hat{f}_{\tau_{1}, \tau_{2}} \mid \mathcal{F}_{t}\right]
\end{gathered}
$$


where we have defined

$$
\begin{gathered}
\hat{f}_{t, s} \equiv \hat{f}_{t}\left(X_{s}, v^{(0)}\left(X_{s}\right), z^{(0)}\left(X_{s}\right)\right) \\
\nabla_{i, s} \equiv \nabla_{i}\left(X_{s}, v^{(0)}\left(X_{s}\right), z^{(0)}\left(X_{s}\right)\right) \\
\gamma_{t} \equiv \gamma\left(X_{t}\right)
\end{gathered}
$$

and $\tau_{1}$ and $\tau_{2}$ are the two interaction times randomly drawn with intensity $\lambda$.

A particle interpretation for the first term is quite simple. A particle $X$ starts at $t$ follows the diffusion (2.3) with (self) interaction intensity $\lambda$. For the first interaction time $\tau_{1}$, it yields $\partial_{v} \hat{f}_{t, \tau_{1}}$ and at the 2 nd interaction time $\tau_{2}$ it yields $\hat{f}_{\tau_{1}, \tau_{2}}$ and decays away. The expectation value can be evaluated by preparing a large number of particles $X$ starting from the same point and obeying the same physical law but spend independent lives. For the second term, the interpretation is more interesting. A particle $X$ starts at time $t$ and follows the diffusion (2.3) with interaction intensity $\lambda$. At the first interaction time $\tau_{1}$, it yields $\partial_{z^{a}} \hat{f}_{t, \tau_{1}}$ and at the same time bears a new particle $Y$. After $\tau_{1}$, the two particles $(X, Y)$ follow the diffusions (2.3) and (2.5), respectively. They have interaction intensity $\lambda$, and at the second interaction point $\tau_{2}$ they yield $\left(Y_{\tau_{1}, \tau_{2}} \gamma\left(X_{\tau_{2}}\right)\right) \nabla_{\tau_{2}} \hat{f}_{\tau_{1}, \tau_{2}}$ and annihilate altogether. As in the first example, the expectation can be calculated by preparing a large number of particle $X$ at the same starting point.

Remark: Note that, if we simply use Eqs. 4.4, 4.21) and the tower property, we have to handle a two-dimensional time integration. It makes naive implementation of Monte Carlo simulation numerically too heavy. In our particle interpretation, this problem is solved by introducing random interaction times with some intensity $\lambda$. One can choose appropriate size of intensity that produces enough amount of events for Monte Carlo simulation.

We now consider an interacting particle interpretation of $Z^{(2)}$. For the evaluation of $Z^{(2)}$, we need to define the second order stochastic flow for $(t<s<u)$ :

$$
\left(\Gamma_{t, s, u}\right)_{j k}^{i}=\frac{\partial^{2}}{\partial x_{t}^{j} \partial x_{s}^{k}} X_{u}^{i}=\frac{\partial}{\partial x_{t}^{j}}\left(Y_{s, u}\right)_{k}^{i}
$$

Since we have

$$
\left(Y_{s, u}\right)_{k}^{i}=\delta_{k}^{i}+\int_{s}^{u}\left(Y_{s, v}\right)_{k}^{l}\left(\partial_{l} \gamma_{0}^{i}\left(X_{v}\right) d v+\partial_{l} \gamma^{i}\left(X_{v}\right) \cdot d W_{v}\right)
$$

it is easy to see that

$$
\begin{aligned}
\left(\Gamma_{t, s, u}\right)_{j, k}^{i}= & \int_{s}^{u}\left(\Gamma_{t, s, v}\right)_{j, k}^{l}\left(\partial_{l} \gamma_{0}^{i}\left(X_{v}\right) d v+\partial_{l} \gamma^{i}\left(X_{v}\right) \cdot d W_{v}\right) \\
& +\int_{s}^{u}\left(Y_{t, v}\right)_{j}^{m}\left(Y_{s, v}\right)_{k}^{l}\left(\partial_{l m} \gamma_{0}^{i}\left(X_{v}\right) d v+\partial_{l m} \gamma^{i}\left(X_{v}\right) \cdot d W_{v}\right)
\end{aligned}
$$

Note that we have $\Gamma_{t, s, s}=0$, regardless of time $s(>t)$. Using the second order stochastic flow, the Malliavin derivative of $Y$ can be written as

$$
\mathcal{D}_{t}^{a}\left(Y_{s, v}\right)_{k}^{i}=\left(\Gamma_{t, s, v}\right)_{j, k}^{i}\left(\gamma^{j}\left(X_{t}\right)\right)_{a}=\left(\Gamma_{t, s, v} \gamma\left(X_{t}\right)\right)_{k, a}^{i}
$$


Consider the process of Malliavin derivative $\mathcal{D}_{t} V_{s}^{(2)}$. One can write its dynamics for $(t<s)$ as

$$
\begin{aligned}
& d\left(\mathcal{D}_{t} V_{s}^{(2)}\right)=-\left(\left(\mathcal{D}_{t} V_{s}^{(1)}\right) \partial_{v}+\left(\mathcal{D}_{t} Z_{s}^{a(1)}\right) \partial_{z^{a}}\right) f\left(X_{s}, v^{(0)}, z^{(0)}\right) d s \\
& \quad-\left(\mathcal{D}_{t} X_{s}^{i}\right)\left\{V_{s}^{(1)} \nabla_{i, s}\left(\partial_{v} f\left(X_{s}, v^{(0)}, z^{(0)}\right)\right)+\left(Z_{s}^{a(1)}\right) \nabla_{i, s}\left(\partial_{z^{a}} f\left(X_{s}, v^{(0)}, z^{(0)}\right)\right)\right\} d s \\
& \quad+\mathcal{D}_{t} Z_{s}^{(2)} \cdot d W_{s} \\
& \mathcal{D}_{t} V_{t}^{(2)}=Z_{t}^{(2)}
\end{aligned}
$$

As before, we define

$$
\widehat{\mathcal{D}_{t} V_{s}^{(2)}}=e^{\int_{t}^{s} \lambda_{u} d u}\left(\mathcal{D}_{t} V_{s}^{(2)}\right)
$$

then its dynamics satisfies the following SDE:

$$
\begin{aligned}
& d\left(\widehat{\mathcal{D}_{t} V_{s}^{(2)}}\right)=\lambda_{s}\left(\widehat{\mathcal{D}_{t} V_{s}^{(2)}}\right) d s-\lambda_{s}\left[\left(\mathcal{D}_{t} X_{s}^{i}\right)\left(V_{s}^{(1)} \nabla_{i, s}\left(\partial_{v} \hat{f}_{t, s}\right)+\left(Z_{s}^{a(1)}\right) \nabla_{i, s}\left(\partial_{z^{a}} \hat{f}_{t, s}\right)\right)\right. \\
& \left.\quad+\left(\left(\mathcal{D}_{t} V_{s}^{(1)}\right) \partial_{v}+\left(\mathcal{D}_{t} Z_{s}^{a(1)}\right) \partial_{z^{a}}\right) \hat{f}_{t, s}\right] d s+e^{\int_{t}^{s} \lambda_{u} d u} \mathcal{D}_{t} Z_{s}^{(2)} \cdot d W_{s} \\
& \left(\widehat{\mathcal{D}_{t} V_{t}^{(2)}}\right)=Z_{t}^{(2)}
\end{aligned}
$$

Then, the same arguments leads to

$$
\begin{aligned}
Z_{t}^{(2)}=\mathbf{1}_{\left\{\tau_{1}>t\right\}} \mathbb{E} & {\left[\mathbf{1}_{\left\{\tau_{1}<T\right\}}\left(\mathcal{D}_{t} X_{\tau_{1}}^{i}\right)\left(V_{\tau_{1}}^{(1)} \nabla_{i, \tau_{1}}\left(\partial_{v} \hat{f}_{t, \tau_{1}}\right)+\left(Z_{\tau_{1}}^{a(1)}\right) \nabla_{i, \tau_{1}}\left(\partial_{z^{a}} \hat{f}_{t, \tau_{1}}\right)\right)\right.} \\
& \left.+\mathbf{1}_{\left\{\tau_{1}<T\right\}}\left(\left(\mathcal{D}_{t} V_{\tau_{1}}^{(1)}\right) \partial_{v}+\left(\mathcal{D}_{t} Z_{\tau_{1}}^{a(1)}\right) \partial_{z^{a}}\right) \hat{f}_{t, \tau_{1}} \mid \mathcal{F}_{t}\right]
\end{aligned}
$$

using the random interaction time $\tau_{1}$.

Proposition $4 Z_{t}^{(2)}$ in (4.22) is equivalently expressed as

$$
\begin{aligned}
& Z_{t}^{a(2)}=\mathbf{1}_{\left\{\tau_{1}>t\right\}} \mathbb{E}\left[\mathbf{1}_{\left\{\tau_{1}<\tau_{2}<T\right\}}\left(Y_{t, \tau_{1}} \gamma_{t}\right)_{a}^{i} \nabla_{i, \tau_{1}}\left(\partial_{v} \hat{f}_{t, \tau_{1}}\right) \hat{f}_{\tau_{1}, \tau_{2}} \mid \mathcal{F}_{t}\right] \\
& +\mathbf{1}_{\left\{\tau_{1}>t\right\}} \mathbb{E}\left[\mathbf{1}_{\left\{\tau_{1}<\tau_{2}<T\right\}}\left(Y_{t, \tau_{1}} \gamma_{t}\right)_{a}^{i} \nabla_{i, \tau_{1}}\left(\partial_{z^{b}} \hat{f}_{t, \tau_{1}}\right)\left(Y_{\tau_{1}, \tau_{2}} \gamma_{\tau_{1}}\right)_{b}^{j} \nabla_{j, \tau_{2}} \hat{f}_{\tau_{1}, \tau_{2}} \mid \mathcal{F}_{t}\right] \\
& +\mathbf{1}_{\left\{\tau_{1}>t\right\}} \mathbb{E}\left[\mathbf{1}_{\left\{\tau_{1}<\tau_{2}<T\right\}}\left(\partial_{v} \hat{f}_{t, \tau_{1}}\right)\left(Y_{t, \tau_{2}} \gamma_{t}\right)_{a}^{i} \nabla_{i, \tau_{2}} \hat{f}_{\tau_{1}, \tau_{2}} \mid \mathcal{F}_{t}\right] \\
& +\mathbf{1}_{\left\{\tau_{1}>t\right\}} \mathbb{E}\left[\mathbf{1}_{\left\{\tau_{1}<\tau_{2}<T\right\}}\left(\partial_{z^{b}} \hat{f}_{t, \tau_{1}}\right)\left(\gamma_{\tau_{1}}\right)_{b}^{j}\left(\Gamma_{t, \tau_{1}, \tau_{2}} \gamma_{t}\right)_{j, a}^{i} \nabla_{i, \tau_{2}} \hat{f}_{\tau_{1}, \tau_{2}} \mid \mathcal{F}_{t}\right] \\
& +\mathbf{1}_{\left\{\tau_{1}>t\right\}} \mathbb{E}\left[\mathbf{1}_{\left\{\tau_{1}<\tau_{2}<T\right\}}\left(\partial_{z^{b}} \hat{f}_{t, \tau_{1}}\right)\left(Y_{t, \tau_{1}} \gamma_{t}\right)_{a}^{j}\left(\partial_{j} \gamma_{\tau_{1}}\right)_{b}^{k}\left(Y_{\tau_{1}, \tau_{2}}\right)_{k}^{i} \nabla_{i, \tau_{2}} \hat{f}_{\tau_{1}, \tau_{2}} \mid \mathcal{F}_{t}\right] \\
& +\mathbf{1}_{\left\{\tau_{1}>t\right\}} \mathbb{E}\left[\mathbf{1}_{\left\{\tau_{1}<\tau_{2}<T\right\}}\left(\partial_{z^{b}} \hat{f}_{t, \tau_{1}}\right)\left(Y_{t, \tau_{2}} \gamma_{t}\right)_{a}^{j}\left(Y_{\tau_{1}, \tau_{2}} \gamma_{\tau_{1}}\right)_{b}^{i} \nabla_{j, \tau_{2}}\left(\nabla_{i, \tau_{2}} \hat{f}_{\tau_{1}, \tau_{2}}\right) \mid \mathcal{F}_{t}\right] .
\end{aligned}
$$

where $\tau_{1}$ and $\tau_{2}$ are sequential interaction times with intensity $\lambda$.

Proof: It can be shown straightforwardly by using the tower property of conditional expectations and commutativity between the indicator functions and the Malliavin derivative due to the independence of $\lambda$. 


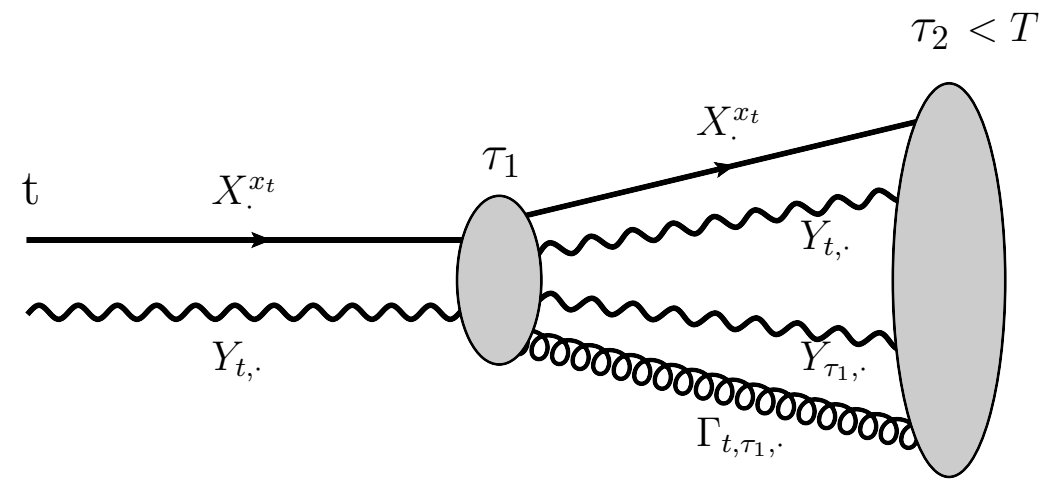

Figure 1: A particle interpretation for $Z_{t}^{(2)}$.

Despite the apparent complexity, required numerical procedures for the evaluation of $Z^{(2)}$ is, in fact, quite simple. We provide a Feynman diagram for the particle interpretation in Figure 1. At the first stage, there are two particles of $\left(X ., Y_{t,}\right)$ with initial values $\left(x_{t},\left\{\delta_{j}^{i}\right\}\right)$, which survive until the second interaction time $\tau_{2}(<T)$. At the first interaction at $\tau_{1}$, two additional particles $\left(Y_{\tau_{1},}, \Gamma_{t, \tau_{1}, .}\right)$ are created. Each interaction occurs randomly with intensity $\lambda$. Note that we already know the initial values of the new particles regardless of the interaction time, which makes numerical simulations possible to carry out. What one has to do is to store the information of $\tau_{1}$ and $\tau_{2}$ and the values of the particles at these times. Then, all the ingredients in expectations can be calculated. Simply repeating independent experiments and taking average will give the desired values.

\section{$4.4 \epsilon$-3rd Order: $V^{(3)}$}

In the similar fashion, we can proceed to higher order. As before, by considering the dynamics of

$$
\hat{V}_{t, s}^{(3)}=e^{\int_{t}^{s} \lambda_{u} d u} V_{s}^{(3)}
$$

one can observe that

$$
\begin{aligned}
V_{t}^{(3)}=\mathbf{1}_{\left\{\tau_{1}>t\right\}} \mathbb{E} & {\left[\mathbf { 1 } _ { \{ \tau _ { 1 } < T \} } \left(V_{\tau_{1}}^{(2)} \partial_{v}+Z_{\tau_{1}}^{a(2)} \partial_{z^{a}}+\frac{1}{2}\left(V_{\tau_{1}}^{(1)}\right)^{2} \partial_{v}^{2}\right.\right.} \\
& \left.\left.+V_{\tau_{1}}^{(1)} Z_{\tau_{1}}^{a(1)} \partial_{v} \partial_{z^{a}}+\frac{1}{2} Z_{\tau_{1}}^{a(1)} Z_{\tau_{1}}^{b(1)} \partial_{z^{a} z^{b}}\right) \hat{f}_{t, \tau_{1}} \mid \mathcal{F}_{t}\right] .
\end{aligned}
$$

It can be written in terms of the fundamental variables simply applying tower property. 
Proposition $5 V_{t}^{(3)}$ can be expressed as

$$
\begin{aligned}
V_{t}^{(3)}= & \mathbf{1}_{\left\{\tau_{1}>t\right\}} \mathbb{E}\left[\mathbf{1}_{\left\{\tau_{1}<\tau_{2}<\tau_{3}\right\}}\left(\partial_{v} \hat{f}_{t, \tau_{1}}\right)\left\{\left(\partial_{v} \hat{f}_{\tau_{1}, \tau_{2}}\right) \hat{f}_{\tau_{2}, \tau_{3}}+\left(\partial_{z^{a}} \hat{f}_{\tau_{1}, \tau_{2}}\right)\left(Y_{\tau_{2}, \tau_{3}} \gamma_{\tau_{2}}\right)_{a}^{i} \nabla_{i, \tau_{3}} \hat{f}_{\tau_{2}, \tau_{3}}\right\}\right. \\
& +\mathbf{1}_{\left\{\tau_{1}<\tau_{2}<\tau_{3}\right\}}\left(\partial_{z^{a}} \hat{f}_{t, \tau_{1}}\right)\left\{\left(Y_{\tau_{1}, \tau_{2}} \gamma_{\tau_{1}}\right)_{a}^{i} \nabla_{i, \tau_{2}}\left(\partial_{v} \hat{f}_{\tau_{1}, \tau_{2}}\right) \hat{f}_{\tau_{2}, \tau_{3}}\right. \\
& +\left(Y_{\tau_{1}, \tau_{2}} \gamma_{\tau_{1}}\right)_{a}^{i} \nabla_{i, \tau_{2}}\left(\partial_{z^{b}} \hat{f}_{\tau_{1}, \tau_{2}}\right)\left(Y_{\tau_{2}, \tau_{3}} \gamma_{\tau_{2}}\right)_{b}^{j} \nabla_{j, \tau_{3}} \hat{f}_{\tau_{2}, \tau_{3}} \\
& +\left(\partial_{z^{b}} \hat{f}_{\tau_{1}, \tau_{2}}\right)\left(\gamma_{\tau_{2}}\right)_{b}^{j}\left(\Gamma_{\tau_{1}, \tau_{2}, \tau_{3}} \gamma_{\tau_{1}}\right)_{j, a}^{i} \nabla_{i, \tau_{3}} \hat{f}_{\tau_{2}, \tau_{3}} \\
& +\left(\partial_{z^{b}} \hat{f}_{\tau_{1}, \tau_{2}}\right)\left(Y_{\tau_{1}, \tau_{2}} \gamma_{\tau_{1}}\right)_{a}^{j}\left(\partial_{j} \gamma_{\tau_{2}}\right)_{b}^{k}\left(Y_{\tau_{2}, \tau_{3}}\right)_{k}^{i} \nabla_{i, \tau_{3}} \hat{f}_{\tau_{2}, \tau_{3}} \\
& \left.\left.+\left(\partial_{z^{b}} \hat{f}_{\tau_{1}, \tau_{2}}\right)\left(Y_{\tau_{1}, \tau_{3}} \gamma_{\tau_{1}}\right)_{a}^{j}\left(Y_{\tau_{2}, \tau_{3}} \gamma_{\tau_{2}}\right)_{b}^{i} \nabla_{j, \tau_{3}}\left(\nabla_{i, \tau_{3}} \hat{f}_{\tau_{2}, \tau_{3}}\right)\right\} \mid \mathcal{F}_{t}\right] \\
+ & \mathbf{1}_{\left\{\tau_{1}>t\right\}} \mathbb{E}\left[\mathbf{1}_{\left\{\tau_{1}<T\right\}} \frac{1}{2}\left(\partial_{v}^{2} \hat{f}_{t, \tau_{1}}\right) \prod_{p=1}^{2}\left(\mathbf{1}_{\left\{\tau_{1}<\tau_{2}^{p}<T\right\}} \hat{f}_{\tau_{1}, \tau_{2}^{p}}\right)\right. \\
& +\mathbf{1}_{\left\{\tau_{1}<T\right\}}\left(\partial_{v} \partial_{z^{a}} \hat{f}_{t, \tau_{1}}\right)\left(\mathbf{1}_{\left\{\tau_{1}<\tau_{2}^{p}<T\right\}} \hat{f}_{\tau_{1}, \tau_{2}^{p}}\right)^{p=1}\left(\mathbf{1}_{\left\{\tau_{1}<\tau_{2}^{p}<T\right\}}\left(Y_{\tau_{1}, \tau_{2}^{p}} \gamma_{\tau_{1}}\right)_{a}^{i} \nabla_{i, \tau_{2}^{p}} \hat{f}_{\tau_{1}, \tau_{2}^{p}}\right)^{p=2} \\
& +\mathbf{1}_{\left\{\tau_{1}<T\right\}} \frac{1}{2}\left(\partial_{z^{a} z^{b}} \hat{f}_{t, \tau_{1}}\right)\left(\mathbf{1}_{\left\{\tau_{1}<\tau_{2}^{p}<T\right\}}\left(Y_{\tau_{1}, \tau_{2}^{p}} \gamma_{\tau_{1}}\right)_{a}^{i} \nabla_{i, \tau_{2}^{p}} \hat{f}_{\tau_{1}, \tau_{2}^{p}}\right)^{p=1} \\
& \left.\times\left(\mathbf{1}_{\left\{\tau_{1}<\tau_{2}^{p}<T\right\}}\left(Y_{\tau_{1}, \tau_{2}^{p}} \gamma_{\tau_{1}}\right)_{b}^{j} \nabla_{j, \tau_{2}^{p}} \hat{f}_{\tau_{1}, \tau_{2}^{p}}\right)^{p=2} \mid \mathcal{F}_{t}\right]
\end{aligned}
$$

where the contents within each bracket of $p \in\{1,2\}$ must be calculated according to the diffusion processes $\left(X^{x_{\tau_{1}}}, Y_{\tau_{1},},\right)_{p=\{1,2\}}$ that follow the identical diffusion laws with the same initial values, but are independent with each other. $\left\{\tau_{i}\right\}_{i \geq 1}$ are sequential random times of interactions drawn with intensity $\lambda .\left\{\tau_{2}^{p}\right\}_{p=1,2}$ should be drawn independently.

Note that, we have introduced two sets of particles labeled by $p \in\{1,2\}$ that follow the same physical laws but perfectly independent with each other to eliminate $\tau_{1}$-conditional expectations. In this way, one can avoid the use of nested Monte Carlo simulation. In Figures 2 and 3, we have provided two Feynman diagrams, one for the first half, and the other for the second half of the expression of $V_{t}^{(3)}$. In simulations, one has to store the interaction times and all the relevant particles values at those points to evaluate the expectations.

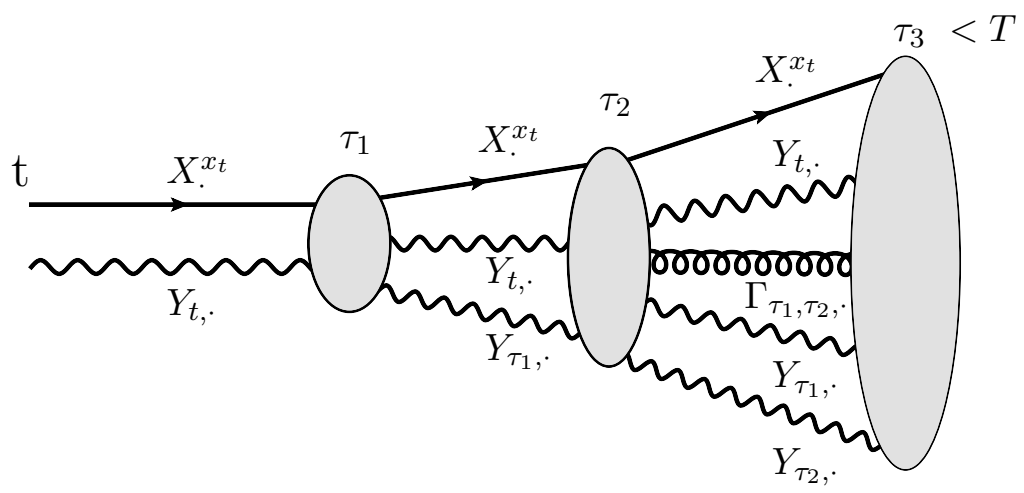

Figure 2: A particle interpretation for the first half of $V_{t}^{(3)}$. 


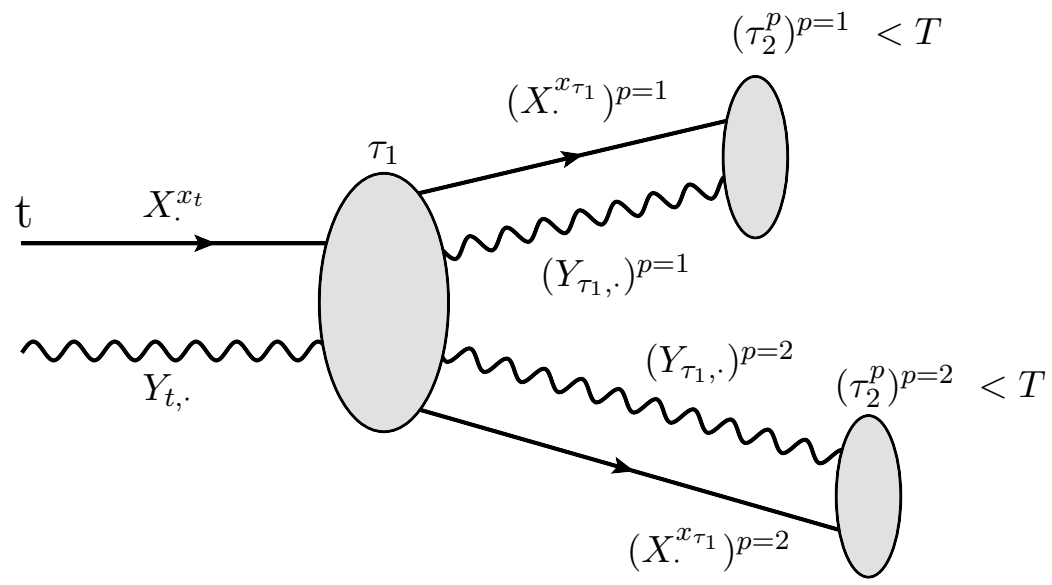

Figure 3: A particle interpretation for the second half of $V_{t}^{(3)}$.

\section{5 $Z^{(3)}$ and $\epsilon$-higher order terms}

The valuation procedures for $Z^{(3)}$ are almost the same as that of $Z^{(2)}$, but we need to introduce a new type of particle corresponding to $\left(\frac{\partial}{\partial x_{t}} \Gamma_{s, u, v,}\right)$. As easily guessed from the previous examples, we need to add one new particle corresponding to a higher order stochastic flow to complete the particle picture at every time when we proceed a $\epsilon$-higher order approximation (of martingale component $Z$ ). A remarkable fact is that all the initial conditions of the new particles created at random times are known beforehand thanks to the characteristics of the Malliavin derivatives. This feature makes one can perform numerical simulations that describe full history of the evolution of particles.

\section{Extension to Fully-Coupled Cases}

We now consider the situation where the underlying state process $X$ also gets the feedbacks from the backward components. By making use of the perturbative technique in PDE framework [12], we shall show that the same strategy in the previous sections works well also in this seemingly much more complicated situation.

The dynamics of whole system is given by

$$
\left\{\begin{array}{l}
d V_{t}=-f\left(t, X_{t}, V_{t}, Z_{t}\right) d t+Z_{t} \cdot d W_{t} \\
V_{T}=\Psi\left(X_{T}\right) \\
d X_{t}=\gamma_{0}\left(t, X_{t}, V_{t}, Z_{t}\right) d t+\gamma\left(t, X_{t}, V_{t}, Z_{t}\right) \cdot d W_{t} \\
X_{0}=x
\end{array}\right.
$$

where we have distinguished time arguments from $X$ to make PDE generator a familiar form. As before, we assume that $V, Z, X$ take value in $\mathbb{R}, \mathbb{R}^{r}$ and $\mathbb{R}^{d}$ respectively, and $W$ denotes a $r$-dimensional Brownian motion. Following the idea of four-step scheme [23, we postulate that $V_{t}$ is given by some appropriate function of $t$ and $X, v(t, X)$. Then it needs 
to satisfy the relevant PDE:

$$
\left\{\begin{aligned}
\partial_{t} v(t, x)+ & \left\{\partial_{i} v(t, x) \gamma_{0}^{i}(t, x, v(t, x), z(t, x))+\frac{1}{2} \partial_{i j} v(t, x)\left(\gamma^{i} \cdot \gamma^{j}\right)(t, x, v(t, x), z(t, x))\right\} \\
& +f(t, x, v(t, x), z(t, x))=0 \\
z(t, x)= & \partial_{i} v(t, x) \gamma^{i}(t, x, v(t, x), z(t, x)) \\
v(T, x)= & \Psi(T, x)
\end{aligned}\right.
$$

The above non-linear PDE cannot be solved in general. Therefore, let us introduce perturbation parameter $\epsilon$ as before,

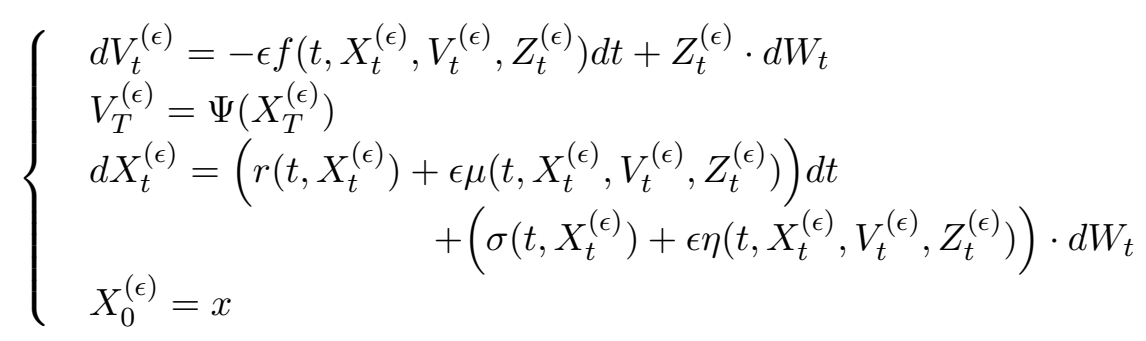

and its corresponding PDE

$$
\left\{\begin{array}{l}
\partial_{i} v^{(\epsilon)}(t, x)+\left\{\partial_{i} v^{(\epsilon)}(t, x) \gamma_{0}^{i}\left(t, x, v^{(\epsilon)}, z^{(\epsilon)}\right)+\frac{1}{2} \partial_{i j} v^{(\epsilon)}(t, x)\left(\gamma^{i} \cdot \gamma^{j}\right)\left(t, x, v^{(\epsilon)}, z^{(\epsilon)}\right)\right\} \\
+\epsilon f\left(t, x, v^{(\epsilon)}, z^{(\epsilon)}\right)=0 \\
z^{(\epsilon)}(t, x)=\partial_{i} v^{(\epsilon)}(t, x) \gamma^{i}\left(t, x, v^{(\epsilon)}, z^{(\epsilon)}\right) \\
v^{(\epsilon)}(T, x)=\Psi(x)
\end{array}\right.
$$

Here, we have extracted the terms free from feedback effects from $X$ 's dynamics 7 :

$$
\left\{\begin{array}{l}
\gamma_{0}\left(t, x, v^{(\epsilon)}, z^{(\epsilon)}\right)=r(t, x)+\epsilon \mu\left(t, x, v^{(\epsilon)}(t, x), z^{(\epsilon)}(t, x)\right) \\
\gamma\left(t, x, v^{(\epsilon)}, z^{(\epsilon)}\right)=\sigma(t, x)+\epsilon \eta\left(t, x, v^{(\epsilon)}(t, x), z^{(\epsilon)}(t, x)\right)
\end{array}\right.
$$

We suppose that the solution of the above PDE can be expanded perturbatively in terms of $\epsilon$ as

$$
\begin{aligned}
& v^{(\epsilon)}(t, x)=v^{(0)}(t, x)+\epsilon v^{(1)}(t, x)+\epsilon^{2} v^{(2)}(t, x)+\cdots \\
& z^{(\epsilon)}(t, x)=z^{(0)}(t, x)+\epsilon z^{(1)}(t, x)+\epsilon^{2} z^{(2)}(t, x)+\cdots
\end{aligned}
$$

As in the previous sections, putting $\epsilon=1$ is expected to give the approximation of the original system as long as the non-linear effects are perturbative.

\subsection{Expansion of non-linear PDE}

Straightforward calculation allows us to expand the original PDE into a series of linear parabolic PDEs. See [12] for details. Firstly, let us define the differential operator $\mathcal{L}$ :

$$
\mathcal{L}(t, x)=r^{i}(t, x) \partial_{i}+\frac{1}{2}\left(\sigma^{i} \cdot \sigma^{j}\right)(t, x) \partial_{i j}
$$

\footnotetext{
${ }^{7}$ Although this can be done somewhat arbitrarily, it may be natural to set $r(t, x)$ and $\sigma(t, x)$ as the expected dynamics of $X$ when all the feedback effects are switched off.
} 
which corresponds to the infinitesimal generator of $X^{(0)}$, ie., the free forward component

$$
\begin{aligned}
d X_{t}^{(0)} & =r\left(t, X_{t}\right) d t+\sigma\left(t, X_{t}\right) \cdot d W_{t} \\
X_{0}^{(0)} & =x
\end{aligned}
$$

Using this generator, we can show that the backward components in each order satisfy:

\section{Zero-th order}

$$
\left\{\begin{array}{l}
\left(\partial_{t}+\mathcal{L}(t, x)\right) v^{(0)}(t, x)=0 \\
v^{(0)}(T, x)=\Psi(x)
\end{array}\right.
$$

and

$$
z^{(0)}(t, x)=\partial_{i} v^{(0)}(t, x) \sigma^{i}(t, x)
$$

Higher expansion order $(n \geq 1)$

$$
\left\{\begin{array}{l}
\left(\partial_{t}+\mathcal{L}(t, x)\right) v^{(n)}(t, x)+G^{(n)}(t, x)=0 \\
v^{(n)}(T, x)=0
\end{array}\right.
$$

where the expression of $G^{(n)}$ and $z^{(n)}$ can be obtained straightforwardly by extracting $\mathcal{O}\left(\epsilon^{n}\right)$ terms from (5.3).

\subsection{Particle Interpretation}

The crucial point in the previous subsection is, because of the perturbation structure in (5.4), the relevant differential operator always derived from $X^{(0)}$ and remains the same for all the expansion orders. In addition, since we put a $\epsilon$-factor in front of the nonlinear terms, $G^{(n)}$ contains the backward components with $\epsilon$-order only up to $(n-1)$. Furthermore, it is clear to see that $z^{(n)}$ can only contain the backward components of $\left\{v^{(m)}\right\}_{\{m \leq n\}}$ and $\left\{z^{(m)}\right\}_{\{m \leq(n-1)\}}$. Therefore, using Feynman-Kac theorem, we see that the PDE in (5.12) is equivalently expressed by

$$
\left\{\begin{array}{l}
d V_{t}^{(n)}=-G^{(n)}\left(t, X_{t}^{(0)}, V_{t}^{(n-1)}, Z_{t}^{(n-1)}, \cdots\right) d t+\tilde{Z}_{t}^{(n)} \cdot d W_{t} \\
V_{T}^{(n)}=0
\end{array}\right.
$$

where the dynamics of the forward component $X^{(0)}$ is already known. Because of the very nature of the perturbative expansion, all the $\left(V^{(m)}, Z^{(m)}\right)_{\{m \geq 1\}}$ appear as a power series and not contained within the non-linear functions. Thus, $V_{t}^{(n)}$ can be solved by the same procedures studied in the previous sections, and also the nice properties of explicitly capped number of branches and interaction points still hold.

Note that $\tilde{Z}_{t}^{(n)}$ is not equal to $Z_{t}^{(n)}$ that contains additional terms through the feedbacks to $X$. However, it is not difficult to calculate these terms. For example, one can observe: 1st order $(n=1)$

$$
\begin{aligned}
G^{(1)}(t, x) & =f^{(0)}(t, x)+\partial_{i} v^{(0)}(t, x) \mu^{i(0)}(t, x)+\partial_{i j} v^{(0)}(t, x)\left(\sigma^{i} \cdot \eta^{j(0)}\right)(t, x) \\
z^{(1)}(t, x) & =\partial_{i} v^{(1)}(t, x) \sigma^{i}(t, x)+\partial_{i} v^{(0)}(t, x) \eta^{i(0)}(t, x)
\end{aligned}
$$


2nd order $(n=2)$

$$
\begin{aligned}
G^{(2)}(t, x)= & \left(v^{(1)}(t, x) \partial_{v}+z^{a(1)}(t, x) \partial_{z^{a}}\right) f^{(0)}(t, x) \\
& +\partial_{i} v^{(1)}(t, x) \mu^{i(0)}(t, x)+\partial_{i} v^{(0)}(t, x)\left(v^{(1)}(t, x) \partial_{v}+z^{a(1)}(t, x) \partial_{z^{a}}\right) \mu^{i(0)}(t, x) \\
& +\partial_{i j} v^{(1)}(t, x)\left(\sigma^{i} \cdot \eta^{j(0)}\right)(t, x)+\frac{1}{2} \partial_{i j} v^{(0)}(t, x)\left(\eta^{i(0)} \cdot \eta^{j(0)}\right)(t, x) \\
& +\partial_{i j} v^{(0)}(t, x) \sigma^{i}(t, x) \cdot\left(v^{(1)}(t, x) \partial_{v}+z^{a(1)}(t, x) \partial_{z^{a}}\right) \eta^{j(0)}(t, x) \\
z^{(2)}(t, x)= & \partial_{i} v^{(2)}(t, x) \sigma^{i}(t, x)+\partial_{i} v^{(1)}(t, x) \eta^{i(0)}(t, x) \\
& +\partial_{i} v^{(0)}(t, x)\left(v^{(1)}(t, x) \partial_{v}+z^{a(1)}(t, x) \partial_{z^{a}}\right) \eta^{i(0)}(t, x)
\end{aligned}
$$

Higher order cases can be obtained similarly.

Let us now consider the particle method to evaluate the relevant terms. Let us fix the initial time as $t$ as before: For the zero-th order, the problem is exactly the same as the decoupled case and we can derive easily $v^{(0)}(t, x)$ and $z^{(0)}(t, x)$ as a function of $x$ by asymptotic expansion 8 . For simplicity, we write $X_{s}^{(0)}$ as $X_{s}$, since the underlying process does not change.

\section{1st order}

As for the first order, observe that $G^{(1)}(t, x)$ is given as an explicit function of $x$ after the completion of the zero-th order calculation. Then, $V_{s}^{(1)}$ follows

$$
\left\{\begin{array}{l}
d V_{s}^{(1)}=-G^{(1)}\left(t, X_{s}\right) d s+\tilde{Z}_{s}^{(1)} \cdot d W_{s} \\
V_{T}^{(1)}=0
\end{array}\right.
$$

and hence, by the same arguments, for $(s>t)$, we have a particle representation as

$$
V_{t}^{(1)}=\mathbf{1}_{\{\tau>t\}} \mathbb{E}\left[\mathbf{1}_{\{\tau<T\}} \hat{G}_{t}^{(1)}\left(\tau, X_{\tau}\right) \mid \mathcal{F}_{t}\right]
$$

where $\hat{G}_{t}^{(1)}$ is defined as

$$
\hat{G}_{t}^{(1)}\left(s, X_{s}\right)=\frac{1}{\lambda_{s}} e^{\int_{t}^{s} \lambda_{u} d u} G^{(1)}\left(s, X_{s}\right)
$$

with some appropriate positive deterministic (or independent) intensity $\lambda$. For martingale component, it is easy to see

$$
Z_{t}^{(1)}=\tilde{Z}_{t}^{(1)}+\partial_{i} v^{(0)}\left(t, x_{t}\right) \eta^{i(0)}\left(t, x_{t}\right)
$$

from (5.15). Here, the particle representation of $\tilde{Z}^{(1)}$ can be derived in the same way as in the decoupled case:

$$
\tilde{Z}_{t}^{a(1)}=\mathbf{1}_{\{\tau>t\}} \mathbb{E}\left[\mathbf{1}_{\{\tau<T\}}\left(Y_{t, \tau} \sigma_{t}\right)_{a}^{i} \partial_{i} \hat{G}_{t}^{(1)}\left(\tau, X_{\tau}\right) \mid \mathcal{F}_{t}\right]
$$

\footnotetext{
${ }^{8}$ As before, this is only to use higher order expansion. For the valuation of the zero-th order itself, one can use the standard Monte Carlo simulation
} 
where $Y_{t, s}(s>t)$ is the stochastic flow of $X$ and is given by

$$
\left(Y_{t, u}\right)_{j}^{i}=\delta_{j}^{i}+\int_{t}^{u}\left(Y_{t, s}\right)_{j}^{k}\left\{\partial_{k} r^{i}\left(s, X_{s}\right) d s+\partial_{k} \sigma^{i}\left(s, X_{s}\right) \cdot d W_{s}\right\}
$$

The second term of $Z^{(1)}$ is already given as an explicit function of $x_{t}$.

\section{2nd order}

We can proceed to higher orders in similar fashion. For the second order, the contribution to $V^{(2)}$ from the first line of $G^{(2)}$ can be calculated in the same way as the decoupled case. Let us consider non-trivial remaining terms. The contribution from $\partial_{i} v^{(1)}(t, x) \mu^{i(0)}(t, x)$, for example, can be calculated as

$$
\begin{aligned}
& \mathbf{1}_{\left\{\tau_{1}>t\right\}} \mathbb{E}\left[\mathbf{1}_{\left\{\tau_{1}<T\right\}} \hat{\mu}_{t}^{i(0)}\left(\tau_{1}, X_{\tau_{1}}\right) \frac{\partial}{\partial x_{\tau_{1}}^{i}}\left(\mathbf{1}_{\left\{\tau_{2}>\tau_{1}\right\}} \mathbb{E}\left[\mathbf{1}_{\left\{\tau_{2}<T\right\}} \hat{G}_{\tau_{1}}^{(1)}\left(\tau_{2}, X_{\tau_{2}}\right) \mid \mathcal{F}_{\tau_{1}}\right]\right) \mid \mathcal{F}_{t}\right] \\
& =\mathbf{1}_{\left\{\tau_{1}>t\right\}} \mathbb{E}\left[\mathbf{1}_{\left\{\tau_{1}<\tau_{2}<T\right\}} \hat{\mu}_{t}^{i(0)}\left(\tau_{1}, X_{\tau_{1}}\right)\left(Y_{\tau_{1}, \tau_{2}}\right)_{i}^{j} \partial_{j} \hat{G}_{\tau_{1}}^{(1)}\left(\tau_{2}, X_{\tau_{2}}\right) \mid \mathcal{F}_{t}\right]
\end{aligned}
$$

where

$$
\hat{\mu}_{t}^{i(0)}\left(s, X_{s}\right)=\frac{1}{\lambda_{s}} e^{\int_{t}^{s} \lambda_{u} d u} \mu^{i(0)}\left(s, X_{s}\right)
$$

Note that the partial derivative of $x$ in $\partial_{i} v^{(1)}\left(\tau_{1}, X_{\tau_{1}}\right)$ should be recognized as the shift of $X$ at the time of $\tau_{1}$, which leads to the first order stochastic flow $Y$ in the above expression.

Next, let us consider the contribution from $\partial_{i j} v^{(1)}(t, x)\left(\sigma^{i} \cdot \eta^{j(0)}\right)(t, x)$. As is the previous example, it is calculated as

$$
\begin{gathered}
\mathbf{1}_{\left\{\tau_{1}>t\right\}} \mathbb{E}\left[\mathbf{1}_{\left\{\tau_{1}<T\right\}}\left(\widehat{\left.\sigma^{i \cdot \eta^{j(0}}\right)}\right)\left(\tau_{1}, X_{\tau_{1}}\right) \frac{\partial^{2}}{\partial x_{\tau_{1}}^{i} \partial x_{\tau_{1}}^{j}}\left(\mathbf{1}_{\left\{\tau_{2}>\tau_{1}\right\}} \mathbb{E}\left[\mathbf{1}_{\left\{\tau_{2}<T\right\}} \hat{G}_{\tau_{1}}^{(1)}\left(\tau_{2}, X_{\tau_{2}}\right) \mid \mathcal{F}_{\tau_{1}}\right]\right) \mid \mathcal{F}_{t}\right] \\
=\mathbf{1}_{\left\{\tau_{1}>t\right\}} \mathbb{E}\left[\mathbf { 1 } _ { \{ \tau _ { 1 } < \tau _ { 2 } < T \} } ( \widehat { \sigma ^ { i } \cdot \eta ^ { j ( 0 ) } } ) ( \tau _ { 1 } , X _ { \tau _ { 1 } } ) \left\{\left(\Gamma_{\tau_{1}, \tau_{2}}\right)_{i j}^{k} \partial_{k} \hat{G}_{\tau_{1}}^{(1)}\left(\tau_{2}, X_{\tau_{2}}\right)\right.\right. \\
\left.\left.+\left(Y_{\tau_{1}, \tau_{2}}\right)_{i}^{k}\left(Y_{\tau_{1}, \tau_{2}}\right)_{j}^{l} \partial_{k l} \hat{G}_{\tau_{1}}^{(1)}\left(\tau_{2}, X_{\tau_{2}}\right)\right\} \mid \mathcal{F}_{t}\right]
\end{gathered}
$$

where $\widehat{\sigma^{i} \cdot \eta^{j(0)}}$ is defined similarly as $\hat{G}^{(1)}$. Note that the second order stochastic flow $\left(\Gamma_{t, s}\right)_{i, j}^{k}$ is defined, for $(u>t)$, as

$$
\left(\Gamma_{t, u}\right)_{i, j}^{k}=\frac{\partial}{\partial x_{t}^{i} \partial x_{t}^{j}}\left(X_{u}^{x_{t}}\right)^{k}
$$

and is given by

$$
\begin{aligned}
\left(\Gamma_{t, u}\right)_{i, j}^{k}= & \int_{t}^{u}\left(\Gamma_{t, s}\right)_{i j}^{l}\left\{\partial_{l} r^{k}\left(s, X_{s}\right) d s+\partial_{l} \sigma^{k}\left(s, X_{s}\right) \cdot d W_{s}\right\} \\
& +\int_{t}^{u}\left(Y_{t, s}\right)_{i}^{l}\left(Y_{t, s}\right)_{j}^{m}\left\{\partial_{l m} r^{k}\left(s, X_{s}\right) d s+\partial_{l m} \sigma^{k}\left(s, X_{s}\right) \cdot d W_{s}\right\}
\end{aligned}
$$

The remaining contributions to $V^{(2)}$ as well as $Z^{(2)}$ can be calculated by the same technique. 
Although tedious calculation is needed, we can proceed to an arbitrary higher order in the same fashion. Note that, also in fully-coupled cases, new particles required in simulation are all derived as stochastic flows of $X$ and hence the initial values at their creations are known beforehand.

\section{Conclusions and Discussions}

In this paper, we have developed an efficient Monte Carlo scheme with an interacting particle representation. It allows straightforward numerical implementation to solve fully non-linear decoupled as well as coupled FBSDEs at each order of perturbative expansion. The appearance of unknown backward components in the expressions of higher order approximations is solved by introducing an appropriate particle interpretation. Although a couple of new particles are created at random interaction times, their initial values are known beforehand. This is due to their properties as the stochastic flows of the underlying sate, which is the crucial point to make straightforward Monte Carlo simulation possible. The proposed method can be applied to semi-linear problems, such as American and Bermudan options, Credit Value Adjustment (CVA), and even fully non-linear issues, such as the optimal portfolio problems in incomplete and/or constrained markets, feedbacks from large investors, and also the analysis of various risk measures. It looks also interesting to use the current method to study higher order FBSDEs, where the higher order Malliavin derivatives exist in the non-linear driver, such as $f\left(t, X_{t}, V_{t}, \mathcal{D}_{t} V, \mathcal{D}_{t}^{2} V\right)$. It can be done straightforwardly by introducing higher order stochastic flows.

Acknowledgment: The authors thank Seisho Sato of the Institute of Statistical Mathematics (ISM) for the helpful discussions about the branching diffusion method.

\section{References}

[1] Bismut, J.M. (1973). "Conjugate Convex Functions in Optimal Stochastic Control," J. Political Econ., 3, 637-654.

[2] Bielecki, T., Rutkowski, M., "Credit Risk: Modeling, Valuation and Hedging," Springer Finance (2002).

[3] Bielecki, T., Jeanblanc, M., Rutkowski, M., "Credit Risk Modeling," Osaka University Press (2009).

[4] Carmona (editor) (2009). "Indifference Pricing," Princeton University Press.

[5] Chakraborty, S., López-Mimbera, J., (2008) "Nonexplosion of a class of semilinear equations via branching particle representation," Adv. Appl. Prob. 40, 250-272.

[6] Crépey, S. (2011). "A BSDE Approach to Counterparty Risk under Funding Constraints," Working paper, Université d'Evry. 
[7] Duffie, D., Huang, M. (1996). "Swap Rates and Credit Quality," Journal of Finance, Vol. 51, No. 3, 921.

[8] Del Moral, P., "Feynman-Kac Formula: Genealogical and Interacting Particle Systems with Applications," Springer (2004)

[9] Doucet A., de Freitas, N., Gordon, N., "Sequential Monte Carlo Methods in Practice," Springer (2001)

[10] El Karoui, N., Peng, S.G., and Quenez, M.C. (1997a). "Backward stochastic differential equations in finance," Math. Finance 7 1-71.

[11] El Karoui, N., Kapoudjian, C., Peng, S.G., and Quenez, M.C. (1997b). "Reflected solutions of backward SDE's, and related obstacle problems for PDE's," the Annals of Probabilities, 25 (2): 702-737.

[12] Fujii, M., and Takahashi, A. (2011). "Analytical Approximation for non-linear FBSDEs with Perturbation Scheme," forthcoming in International Journal of Theoretical and Applied Finance.

[13] Fujii, M., and Takahashi, A. (2012), "Perturbative Expansion of FBSDE in an Incomplete Market with Stochastic Volatility," CARF working paper series, CARF-F-270, available at ssrn and arXiv.

[14] Fujii, M., Sato, S., Takahashi A.: In preparation.

[15] Fujita, H., "On the blowing up of solutions of the Cauchy problem for $u_{t}=\Delta u+$ $u^{1+\alpha}, "$ J. Fac. Sci. Univ. Tokyo 13 (1966), 109-124

[16] Henry-Labordère, P., "Counterparty Risk Valuation: A marked branching diffusion approach", arXiv:1203.2369

[17] Ikeda, N., Nagasawa, M., and Watanabe, S. (1965), "Branching Markov processes," Abstracts Proc. Japan Acad. 41, 816-821.

[18] Ikeda, N., Nagasawa, M., and Watanabe, S. (1966), "Branching Markov processes," Abstracts Proc. Japan Acad. 42, 252-257, 370-375, 380-384, 719-724, 1016-1021, 1022 1026 .

[19] Ikeda, N., Nagasawa, M., and Watanabe, S., (1968), "Branching Markov processes I(II)," J. Math. Kyoto Univ. 8, 233-278, 365-410.

[20] Ikeda, N. et.al., (1996, 1997) "Seminar on Probability," vol:23 I-II and vol:25 I-II.

[21] Kunitomo, N. and Takahashi, A. (2003). "On Validity of the Asymptotic Expansion Approach in Contingent Claim Analysis," Annals of Applied Probability, 13, No.3, 914-952.

[22] Labart, C., Lelong, J., (2011), "A parallel algorithm for solving BSDEs-application to pricing and hedging of American options," available at arXiv:1102.4666. 
[23] Ma, J., Protter, P., and Yong, J., 1994, "Solving forward-backward stochastic differential equations explicitly", Prob.\& Related Fields, 98.

[24] Ma, J., and Yong, J. (2000), "Forward-Backward Stochastic Differential Equations and their Applications," Springer.

[25] Nagasawa, M., Sirao, T., (1969), "Probabilistic Treatment of the Blowing up of Solutions for a Nonlinear Integral Equation," Trans. Amer. Math. Soc. Vol 139, 301-310.

[26] McKean, H., P., "Application of Brownian Motion to the Equation of KolmogorovPetrovskii-Piskunov," Communications on Pure and Applied Mathematics, Vol. XXVIII, 323-331 (1975).

[27] Pardoux, E., and Peng, S. (1990). "Adapted Solution of a Backward Stochastic Differential Equation," Systems Control Lett., 14, 55-61.

[28] Takahashi, A. (1999). "An Asymptotic Expansion Approach to Pricing Contingent Claims," Asia-Pacific Financial Markets, 6, 115-151.

[29] Takahashi, A., Takehara, K., and Toda, M. (2011). "A General Computation Scheme for a High-Order Asymptotic Expansion Method," CARF Working Paper F-242, available at http://www.carf.e.u-tokyo.ac.jp/workingpaper/.

[30] Takahashi, A. and Yoshida, N. (2004). "An Asymptotic Expansion Scheme for Optimal Investment Problems," Statistical Inference for Stochastic Processes, 7, No.2, $153-188$. 